\title{
Community-acquired pneumonia and survival of critically ill acute exacerbation of COPD patients in respiratory intensive care units
}

This article was published in the following Dove Press journal:

International Journal of COPD

9 August 2016

Number of times this article has been viewed

\author{
Zhiwei Lu* \\ Yusheng Cheng* \\ Xiongwen Tu \\ Liang Chen \\ Hu Chen \\ Jian Yang \\ Jinyan Wang \\ Liqin Zhang
}

Department of Respiratory Medicine, Yijishan Hospital of Wannan Medical College, Wuhu, People's Republic of China

*These authors contributed equally to this work
Correspondence: Yusheng Cheng Department of Respiratory Medicine, Yijishan Hospital of Wannan Medical College, 2 Zeshan West Road, 24I00I Wuhu, People's Republic of China Tel +865535739006 Email chengys|222@I26.com
Purpose: The aim of this study was to appraise the effect of community-acquired pneumonia (CAP) on inhospital mortality in critically ill acute exacerbation of COPD (AECOPD) patients admitted to a respiratory intensive care unit.

Patients and methods: A retrospective observational study was performed. Consecutive critically ill AECOPD patients receiving treatment in a respiratory intensive care unit were reviewed from September 1, 2012, to August 31, 2015. Categorical variables were analyzed using chi-square tests, and continuous variables were analyzed by Mann-Whitney $U$-test. Kaplan-Meier analysis was used to assess the association of CAP with survival of critically ill AECOPD patients for univariate analysis. Cox's proportional hazards regression model was performed to identify risk factors for multivariate analysis.

Results: A total of 80 consecutive eligible individuals were reviewed. These included 38 patients with CAP and 42 patients without CAP. Patients with CAP had a higher inhospital rate of mortality than patients without CAP $(42 \%$ vs $33.3 \%, P<0.05)$. Kaplan-Meier survival analysis showed that patients with $\mathrm{CAP}$ had a worse survival rate than patients without CAP $(P<0.05)$. Clinical characteristics, including Acute Physiology and Chronic Health Evaluation II (APACHE II) score, C-reactive protein, and CAP, were found to be closely associated with survival of AECOPD individuals. Further multivariate Cox regression analysis confirmed that CAP and APACHE II were independent risk factors for inhospital mortality in critically ill AECOPD patients (CAP: hazard ratio, 5.29; 95\% CI, 1.50-18.47, $P<0.01$ and APACHE II: hazard ratio, $1.20 ; 95 \% \mathrm{CI}, 1.06-1.37, P<0.01)$.

Conclusion: CAP may be an independent risk factor for higher inhospital mortality in critically ill AECOPD patients.

Keywords: community-acquired pneumonia, AECOPD, respiratory intensive care unit, risk factor, mortality, critically ill

\section{Introduction}

COPD is an aggressive disease, acknowledged as the fourth leading cause of death among chronic diseases. It poses a huge public health burden worldwide, even though it is preventable and treatable. Several common clinical complications have been reported to share close associations with the poor outcome in COPD patients, such as cardiovascular disease, lung cancer, and infection. ${ }^{1}$ Acute exacerbation of COPD (AECOPD) is characterized by acute changes in clinical symptoms of COPD beyond normal day-to-day variation requiring emergency medical intervention. Patients with severe exacerbations should be transferred to respiratory disease wards to receive further 
treatment. Patients with acute respiratory failure or septic shock may need ventilator support or intensive care. ${ }^{1}$

Pneumonia is reported to be a major contributor to hospitalization for AECOPD and shares a close relationship with poor patient outcomes. Moreover, patients with pneumonic exacerbation have been found to be admitted into intensive care units (ICUs) more often and stay there longer than those with nonpneumonic exacerbations. ${ }^{2}$ It is well established that administration of corticosteroids has a beneficial effect on severe AECOPD patients. However, they also increase the risk of pneumonia. ${ }^{3,4}$ In recent years, mounting evidence has indicated that community-acquired pneumonia (CAP), which impairs lung defense, is reported to be one of the more common reasons for admission into ICUs and to affect outcomes of COPD patients. ${ }^{5}$ A previous study reported inhospital mortality in COPD patients complicated by CAP to be $12.2 \%{ }^{6}$ Pneumonia also predicts higher mortality in COPD patients with repeated exacerbation events. ${ }^{2}$

However, few studies have investigated the effect of CAP on inhospital mortality in critical AECOPD patients hospitalized in respiratory ICUs (RICUs). Given the relationship between CAP and survival of patients with critical AECOPD remains largely undetermined, a retrospective observational study was conducted to appraise the effects of CAP inhospital mortality in critically ill AECOPD patients.

\section{Patients and methods}

The study protocol acquired approval from the Research Ethics Committee of Yijishan Hospital of Wannan Medical College. Written informed consent was obtained from all participants or their relatives. This retrospective observational study was performed in a single RICU of a tertiary teaching hospital. Consecutive critical AECOPD patients hospitalized in the RICU were reviewed from September 1, 2012, to August 31, 2015.

AECOPD was defined as an event characterized by acute changes in clinical symptoms beyond normal day-to-day variation according to the criteria in current guidelines. ${ }^{1}$ COPD patient categories were as follows: subgroup A, low risk and few symptoms; subgroup $\mathrm{B}$, low risk and more symptoms; subgroup $\mathrm{C}$, high risk and few symptoms; subgroup D, high risk and more symptoms. More details were described previously. ${ }^{1}$ The diagnostic criteria of CAP are as follows: 1) symptoms of an acute lower respiratory tract illness (coughing and at least one other lower respiratory tract symptom); 2) new focal chest signs upon examination, at least one systemic feature (a symptom complex of sweating, fevers, shivers, aches and pains, temperature of $\geq 38^{\circ} \mathrm{C}$, or combination of these); 3) new radiographic shadowing for which there was no other explanation; and 4) no other explanation for the illness, which was treated as CAP with antibiotics. ${ }^{7}$ Acute Physiology and Chronic Health Evaluation II (APACHE II) scores were used to assess the severity of patients requiring intensive care on admission, as detailed in a previous work. ${ }^{8}$ Noninvasive ventilation (NIV) treatment failure was defined as oxygenation and clinical conditions that could not be improved or patients who needed emergency endotracheal intubation while receiving NIV. ${ }^{9}$ Demographic details, comorbid conditions, and laboratory measurements were also collected.

All reviewed patients underwent pulmonary computed tomography before transfer to RICU. Collectively, patients with a diagnosis of critical AEOPD and hospitalized in the RICU were eligible for inclusion in this study. Patients younger than 45 years or older than 85 years, length of stay $>30$ days or $<2$ days, receiving palliative care only, health care-associated pneumonia, septic shock, severe left heart failure, pulmonary embolism, diffuse pulmonary fibrosis disease, renal replacement therapy, and asthma were not included. The clinical data from patients undergoing their first RICU admission were collected.

\section{Statistical analysis}

Continuous data are presented as mean $\pm \mathrm{SD}$, and categorical data are presented as number (n) or percentage. Continuous variables were analyzed using the Mann-Whitney $U$-test, and categorical variables were analyzed by chi-square test for nonparametric variables. A Kaplan-Meier survival curve was used to analyze the effect of CAP on survival of critically ill AECOPD patients hospitalized in the RICU. A log-rank test was used to evaluate statistical differences in the survival curves. Cox's proportional hazards regression model analysis was performed to assess risk factors for inhospital mortality for multivariate analysis. $P<0.05$ was considered statistically significant. The statistical analyses were performed using a software package (SPSS for Windows, release 22.0; IBM Corporation, Armonk, NY, USA).

\section{Results}

Consistent with inclusion and exclusion criteria, a total of 80 consecutive cases (58 men and 22 women) with critical AECOPD admitted to RICU were reviewed during this 2-year study. All cases were divided into a CAP group (38 cases) and no-CAP group (42 cases). The baseline characteristics of the two groups are given in Table 1. The levels of arterial blood $\mathrm{PaCO}_{2}$ were significantly lower in cases with CAP than in cases without CAP, with $74.22 \pm 30.81 \mathrm{mmHg}$, and $86.54 \pm 26.38 \mathrm{mmHg}$, respectively $(P<0.05)$. A significant 
Table I Demographic data of AECOPD patients with and without CAP

\begin{tabular}{|c|c|c|c|}
\hline Characteristics & $\begin{array}{l}\text { AECOPD } \\
\text { with CAP }\end{array}$ & $\begin{array}{l}\text { AECOPD } \\
\text { without CAP }\end{array}$ & $P$-value \\
\hline Subjects (n) & 38 & 42 & \\
\hline Males (n) & 28 & 30 & 1.000 \\
\hline Age (years) & $74.11 \pm 8.02$ & $75.36 \pm 8.23$ & 0.559 \\
\hline COPD categories (n) & & & 0.19 \\
\hline$B$ subgroup & 6 & 14 & \\
\hline C subgroup & 18 & 15 & \\
\hline D subgroup & 14 & 13 & \\
\hline $\mathrm{pH}$ value & $7.28 \pm 0.16$ & $7.27 \pm 0.11$ & 0.335 \\
\hline $\mathrm{PaO}_{2} / \mathrm{FiO}_{2}$ & $224.90 \pm 86.69$ & $223.27 \pm 86.11$ & 0.933 \\
\hline $\mathrm{PaCO}_{2}(\mathrm{mmHg})$ & $74.22 \pm 30.81$ & $86.54 \pm 26.38$ & 0.037 \\
\hline Blood lactate (mmol/L) & $1.55 \pm 1.07$ & $1.30 \pm 0.66$ & 0.420 \\
\hline Diabetes (n) & 2 & 2 & 1.000 \\
\hline Hypertension (n) & 13 & 15 & 1.000 \\
\hline APACHE II score & $|8.53 \pm 5.7|$ & $18.55 \pm 3.77$ & 0.274 \\
\hline White blood cells $\left(\times 10^{9} / L\right)$ & $9.80 \pm 4.61$ & $10.39 \pm 5.84$ & 0.874 \\
\hline CRP (mg/L) & $43.53 \pm 52.16$ & $49.06 \pm 86.48$ & 0.179 \\
\hline NIV failure (n) & 16 & 15 & 0.648 \\
\hline Use of glucocorticoid & 14 & 18 & 0.651 \\
\hline Length of stay (n) & $9.80 \pm 4.61$ & $|I .3| \pm 5.03$ & 0.204 \\
\hline Inhospital death (n) & 16 & 8 & 0.030 \\
\hline
\end{tabular}

Notes: Data are given as mean \pm standard deviation, or $n$. Bold represents statistically significant.

Abbreviations: AECOPD, acute exacerbation of COPD; APACHE II, Acute Physiology and Chronic Health Evaluation II; CAP, community-acquired pneumonia; CRP, C-reactive protein; NIV, noninvasive ventilation.

difference in inhospital mortality was observed between cases with and without CAP $(20 \%$ vs $10 \%, P<0.05)$. Kaplan-Meier survival curve analysis showed the survival time of cases with CAP to be significantly shorter than that of cases without CAP during hospitalization period (odd ratio, $2.82,95 \%$ CI, $1.22-6.50, P<0.05)$, as indicated in Figure 1.

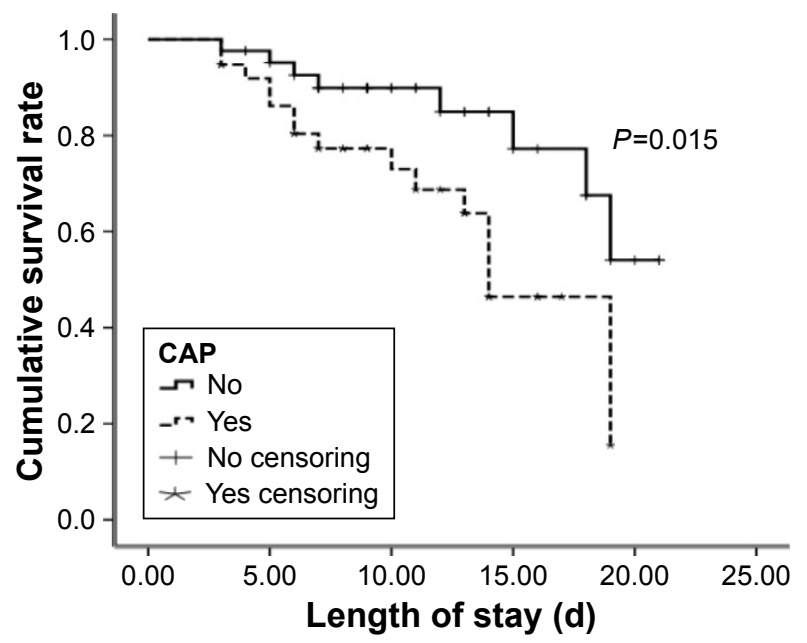

Figure I Comparisons of survival time of critically ill AECOPD patients with and without CAP in RICU.

Note: Kaplan-Meier survival curve analysis was performed, and log-rank test was used; $P<0.05$ was considered statistically significant.

Abbreviations: AECOPD, acute exacerbation of COPD; CAP, community-acquired pneumonia; RICU, respiratory intensive care unit.
However, other clinical characteristics, including sex, age, COPD patient categories, $\mathrm{pH}$ value, incidences of diabetes and hypertension, APACHE II scale, white blood cell counts, $\mathrm{PaO}_{2} / \mathrm{FiO}_{2}$, blood lactate and $\mathrm{C}$-reactive protein (CRP), rates of NIV treatment failure, length of glucocorticoid use over 5 days, and length of stay, showed no statistical differences between cases with and without CAP $(P>0.05)$.

Table 2 shows comparisons of clinical characteristics of survivals and nonsurvivals among critically ill AECOPD patients. Here, 22 of the survivals and 16 of the nonsurvivals were complicated by CAP $(39.5 \%$ vs $66.7 \%, P<0.05)$. Nonsurvivals had higher APACHE II scores than survivals $(21.10 \pm 6.22$ vs $17.52 \pm 3.55, P<0.05)$. Similarly, greater increases in the CRP level were observed in nonsurvivals than in survivals $(49.37 \pm 45.28 \mathrm{mg} / \mathrm{L}$ vs $45.17 \pm 80.97 \mathrm{mg} / \mathrm{L}$, $P<0.05)$. However, there were no significant differences in other characteristics $(P>0.05)$.

Independent risk factors of inhospital mortality in critically ill AECOPD patients were assessed using Cox's proportional hazards regression model analysis and are displayed in Table 3. Independent risk factors for increased inhospital mortality in critically ill AECOPD patients admitted to RICU were as follows: complicated by CAP (hazard ratio, 5.007; 95\% CI, 1.658-15.542, $P<0.01)$ and with a high APACHE II score (hazard ratio, 1.173; 95\% CI, 1.024-1.343,

Table 2 Clinical characteristics regarding survival and nonsurvival in critical AECOPD patients

\begin{tabular}{|c|c|c|c|}
\hline Characteristics & Survivals & Nonsurvivals & $P$-value \\
\hline Subjects (n) & 56 & 24 & \\
\hline Males (n) & 41 & 17 & 1.000 \\
\hline Age (years) & $74.82 \pm 7.46$ & $74.63 \pm 9.63$ & 0.756 \\
\hline COPD categories (n) & & & 0.58 \\
\hline B subgroup & 15 & 5 & \\
\hline C subgroup & 21 & 12 & \\
\hline D subgroup & 20 & 7 & \\
\hline $\mathrm{pH}$ value & $7.28 \pm 0.14$ & $7.27 \pm 0.13$ & 0.661 \\
\hline $\mathrm{PaO}_{2} / \mathrm{FiO}_{2}$ & $219.91 \pm 78.98$ & $229.79 \pm 88.73$ & 0.371 \\
\hline $\mathrm{PaCO}_{2}(\mathrm{mmHg})$ & $80.15 \pm 30.46$ & $8 I .4 I \pm 26.38$ & 0.833 \\
\hline Blood lactate $(\mathrm{mmol} / \mathrm{L})$ & $1.43 \pm 0.94$ & $\mathrm{I} .4 \mathrm{I} \pm 0.73$ & 0.736 \\
\hline Diabetes (n) & 4 & 0 & 0.311 \\
\hline Hypertension (n) & 23 & 5 & 0.124 \\
\hline APACHE II score & $17.52 \pm 3.55$ & $21.10 \pm 6.22$ & 0.031 \\
\hline White blood cells $\left(\times 10^{9} / \mathrm{L}\right)$ & $10.14 \pm 5.66$ & $10.04 \pm 4.34$ & $0.6 \mathrm{II}$ \\
\hline CRP (mg/L) & $45.17 \pm 80.97$ & $49.37 \pm 45.28$ & 0.039 \\
\hline NIV failure (n) & 20 & II & 0.457 \\
\hline Use of glucocorticoid & 20 & 12 & 0.320 \\
\hline Length of stay (n) & $10.93 \pm 4.78$ & $9.92 \pm 5.59$ & 0.315 \\
\hline With CAP (n) & 22 & 16 & 0.03 \\
\hline
\end{tabular}

Notes: Data are given as mean \pm standard deviation, or $n$. Bold represents statistically significant.

Abbreviations: AECOPD, acute exacerbation of COPD; APACHE II, Acute Physiology and Chronic Health Evaluation II; CAP, community-acquired pneumonia; CRP, C-reactive protein; NIV, noninvasive ventilation. 
Table 3 Independent risk factors for inhospital mortality as indicated by Cox's proportional hazards regression model analysis

\begin{tabular}{llll}
\hline Variable & OR & $\mathbf{9 5 \% ~ C l}$ & P-value \\
\hline CAP & 5.077 & $1.658-15.542$ & $\mathbf{0 . 0 0 4}$ \\
$\mathrm{CRP}$ & 1.00 & $0.99-1.009$ & 0.808 \\
$\mathrm{NIV}$ failure & 0.53 & $0.058-1.614$ & 0.163 \\
$\mathrm{PaO}_{2} / \mathrm{FiO}_{2}$ & 0.997 & $0.991-1.004$ & 0.424 \\
$\mathrm{Glucocorticoid} \mathrm{use}_{\text {Blood lactate }}$ & 0.969 & $0.342-2.748$ & 0.953 \\
APACHE II score & 1.143 & $0.55-2.372$ & 0.721 \\
\hline
\end{tabular}

Note: Bold represents statistically significant.

Abbreviations: APACHE II, Acute Physiology and Chronic Health Evaluation II; $\mathrm{CAP}$, community-acquired pneumonia; $\mathrm{Cl}$, confidence interval; CRP, C-reactive protein; NIV, noninvasive ventilation; OR, odds ratio.

$P<0.01)$. Levels of CRP, NIV treatment failure, $\mathrm{PaO}_{2} / \mathrm{FiO}_{2}$, glucocorticoid use, and blood lactate were not found to increase inhospital mortality in those patients.

\section{Discussion}

CAP is a frequent comorbidity or overlap disease in AECOPD patients who require hospitalization. ${ }^{6,10} \mathrm{~A}$ previous analysis indicated that the incidence of pneumonia in elderly COPD patients was 54.2/1,000 person-years, which was found to be more common in COPD patients in the US than in those from other countries. ${ }^{11}$ Several studies have identified factors predisposing COPD patients to CAP, such as disease severity, old age, long-term use of inhaled corticosteroids, and dysphasia. ${ }^{3,12-14}$ The inhospital mortality rate of pneumonic AECOPD has been reported to be significantly higher than that of nonpneumonic exacerbations. ${ }^{15,16}$ In this way, CAP is a valuable predictive factor of poor prognosis in AECOPD patients. Use of inhaled corticosteroids alone or in combination with a long-acting $\beta 2$-agonist increases the risk of pneumonia in COPD patients, but the overall mortality is not affected. ${ }^{3,13,14}$ AECOPD patients with pneumonia were found to use NIV more frequently and remain hospitalized longer, but they were not found to have significantly higher rates of inhospital mortality than those without pneumonia. ${ }^{17}$

In the present study, critically ill AECOPD patients with CAP had higher inhospital mortality (20\%), than individuals without CAP, who had $10 \%$. However, Takir et al ${ }^{5}$ reported that inhospital mortality in COPD patients with pneumonia in the ICU was $12.5 \%$. Many factors may account for these inconsistent results. First, the mean age of patients in the current study was higher than that in the previous study; second, the inclusion and exclusion criteria were quite different; and finally, the medical facilities and similar factors may also have caused some differences.

The current study also showed the level of arterial blood $\mathrm{PaCO}_{2}$ to be lower in cases with CAP than in cases without CAP.
The level of arterial blood $\mathrm{PaCO}_{2}$ in patients with lung injuries is often lower than normal. This interferes with ventilation-perfusion matching and gas exchange in the respiratory system through complicated mechanisms. ${ }^{18}$ Hypercapnia also attenuates several inflammatory processes, which may protect organs from stress injuries. ${ }^{19}$ In this way, permissive hypercapnia may benefit critically ill AECOPD patients. However, in the present study, the level of arterial blood $\mathrm{PaCO}_{2}$ was not significantly different between survivals and nonsurvivals. The hypercapnia observed in these patients may offset its beneficial effects.

NIV, systemic corticosteroid treatment, and shorter ICU stays were found to be associated with reduced mortality in COPD patients with CAP, as indicated by data from a study performed on the ICUs of 19 different hospitals in Turkey from October 2008 to January $2011 .{ }^{20}$ However, this study excluded patients with COPD exacerbation from further analysis. A large body of evidence has shown that the use of NIV benefits AECOPD patients and decreases both the need for invasive ventilation and inhospital mortality. ${ }^{21,22}$ Unlike in AECOPD patients with acute respiratory failure, NIV treatment was not very beneficial to patients with acute respiratory failure due to pneumonia, which has limited its clinical usage..$^{23}$ In the present study, results showed that NIV intervene did not provide additional benefits for critically ill AECOPD individuals with CAP. For this reason, NIV treatment should be recommended to alleviate respiratory distress syndrome in select critically ill AECOPD patients with pneumonia rather than in critical AECOPD patients generally. ${ }^{24}$

Systemic corticosteroid treatment shares close association with lower rates of mortality in patients with $\mathrm{CAP} .{ }^{25}$ However, it was not found to significantly change rates of mortality in AECOPD patients requiring intensive care, though it may be associated with a significant increase in the success of noninvasive mechanical ventilation and a reduction in the duration of mechanical ventilation. ${ }^{26,27}$ However, the beneficial effects of corticosteroids were only observed in noncritically ill AECOPD patients and not in critically ill AECOPD patients, regardless of whether ventilation was invasive or noninvasive. ${ }^{27}$ As in previous reports, the results of the current study also indicated that critically ill AECOPD individuals with CAP did not benefit from systemic corticosteroid administration. In this way, systemic corticosteroid treatment may not be suitable for critical AECOPD patients with CAP.

The APACHE II score is an independent factor associated with mortality in AECOPD patients admitted to RICU. ${ }^{28}$ In the current study, APACHE II scores and the level of CRP were significantly higher in nonsurvivals than in survivals. 
By multivariate analysis, only CAP and APACHE II scores were found to be independently associated with an increased mortality in AECOPD patients, and CRP, NIV, and glucocorticoid use lasting $>5$ days were not.

The present work reveals the disadvantage that CAP places on critical AECOPD patients who are hospitalized in RICUs. Nevertheless, this study still has several limitations that may have influenced its results. First, it was a retrospective study performed in a single RICU. This issue merits further multicenter prospective studies in the future. Second, the results of this study are applicable to only critical AECOPD patients and do not apply even to AECOPD patients whose condition is not critical. Finally, the sample size was small, although all reasonable efforts were made to minimize error and bias.

\section{Conclusion}

The results of the present study suggest that CAP is an independent risk factor for increased inhospital mortality in critical AECOPD patients. The close association between CAP and AECOPD warrants further investigation.

\section{Acknowledgments}

Ren-guang Pei, Department of Interventional Therapy, Yijishan Hospital of Wannan Medical College, provided valuable insight during the revision of this article. Support for this study has been awarded to ZL by the Talent Introduction Procedure (no YR201107) of Wannan Medical College and to YC by the Natural Science Foundation of Wannan Medical College for Middle-aged and Young Scientific Researchers (no WK2014F43). No author has any relevant financial relationship.

\section{Author contributions}

All authors performed critical revisions of the article and approved the final version for publication. YC contributed to study concept and design, interpretation, drafting of the article, and study supervision. ZL contributed to statistical analysis, interpretation, and drafting of the manuscript. XT, LZ, LC, HC, JW, and JY contributed to data collection.

\section{Disclosure}

The authors report no conflicts of interest in this work.

\section{References}

1. Global Initiative for Chronic Obstructive Lung Disease (GOLD) [homepage on the Internet]. The Global Strategy for the Diagnosis, Management and Prevention of COPD. 2015. Available from: http:// www.goldcopd.org/.
2. Søgaard M, Madsen M, Løkke A, Hilberg O, Sørensen HT, Thomsen RW. Incidence and outcomes of patients hospitalized with COPD exacerbation with and without pneumonia. Int J Chron Obstruct Pulmon Dis. 2016;11:455-465.

3. Festic E, Scanlon PD. Incident pneumonia and mortality in patients with chronic obstructive pulmonary disease. A double effect of inhaled corticosteroids? Am J Respir Crit Care Med. 2015;191:141-148.

4. Lee $\mathrm{MC}$, Lee $\mathrm{CH}$, Chien SC, et al. Inhaled corticosteroids increase the risk of pneumonia in patients with chronic obstructive pulmonary disease: a nationwide cohort study. Medicine (Baltimore). 2015;94:e1723.

5. Takir HB, Karakurt Z, Salturk C, et al. Reasons for ICU demand and long-term follow-up of a chronic obstructive pulmonary disease cohort. COPD. 2014;11:627-638

6. Yamauchi Y, Yasunaga H, Matsui H, et al. Comparison of clinical characteristics and outcomes between aspiration pneumonia and community-acquired pneumonia in patients with chronic obstructive pulmonary disease. BMC Pulm Med. 2015;15:69.

7. Lim WS, Baudouin SV, George RC, et al; Pneumonia Guidelines Committee of the BTS Standards of Care Committee. BTS guidelines for the management of community acquired pneumonia in adults: update 2009. Thorax. 2009;64(suppl 3):iii1-iii55.

8. Knaus WA, Draper EA, Wagner DP, Zimmerman JE. APACHE II: a severity of disease classification system. Crit Care Med. 1985;13: $818-829$.

9. Antonelli M, Conti G, Bufi M, et al. Noninvasive ventilation for treatment of acute respiratory failure in patients undergoing solid organ transplantation: a randomized trial. JAMA. 2000;283:235-241.

10. Boixeda R, Bacca S, Elias L, et al. Pneumonia as comorbidity in chronic obstructive pulmonary disease (COPD). Differences between acute exacerbation of COPD and pneumonia in patients with COPD. Arch Bronconeumol. 2014;50:514-520.

11. Ryan M, Suaya JA, Chapman JD, Stason WB, Shepard DS, Thomas CP. Incidence and cost of pneumonia in older adults with COPD in the United States. PLoS One. 2013;8:e75887.

12. Müllerova H, Chigbo C, Hagan GW, et al. The natural history of community-acquired pneumonia in COPD patients: a population database analysis. Respir Med. 2012;106:1124-1133.

13. Kew KM, Seniukovich A. Inhaled steroids and risk of pneumonia for chronic obstructive pulmonary disease. Cochrane Database Syst Rev. 2014;3:CD010115.

14. Singanayagam A, Chalmers JD, Akram AR, Hill AT. Impact of inhaled corticosteroid use on outcome in COPD patients admitted with pneumonia. Eur Respir J. 2011;38:36-41.

15. Steer J, Norman EM, Afolabi OA, Gibson GJ, Bourke SC. Dyspnoea severity and pneumonia as predictors of in-hospital mortality and early readmission in acute exacerbations of COPD. Thorax. 2012;67: $117-121$.

16. Myint PK, Lowe D, Stone RA, Buckingham RJ, Roberts CM. U.K. National COPD Resources and Outcomes Project 2008: patients with chronic obstructive pulmonary disease exacerbations who present with radiological pneumonia have worse outcome compared to those with non-pneumonic chronic obstructive pulmonary disease exacerbations. Respiration. 2011;82:320-327.

17. Andreassen SL, Liaaen ED, Stenfors N, Henriksen AH. Impact of pneumonia on hospitalizations due to acute exacerbations of COPD. Clin Respir J. 2014;8:93-99.

18. Curley G, Laffey JG, Kavanagh BP. Bench-to-bedside review: carbon dioxide. Crit Care. 2010;14:220.

19. Laffey JG, Kavanagh BP. Carbon dioxide and the critically ill - too little of a good thing? Lancet. 1999;354:1283-1286.

20. Cilli A, Erdem H, Karakurt Z, et al. Community-acquired pneumonia in patients with chronic obstructive pulmonary disease requiring admission to the intensive care unit: risk factors for mortality. J Crit Care. 2013;28:975-979.

21. Dres M, Tran TC, Aegerter P, et al. Influence of ICU case-volume on the management and hospital outcomes of acute exacerbations of chronic obstructive pulmonary disease. Crit Care Med. 2013;41:1884-1892. 
22. Lindenauer PK, Stefan MS, Shieh MS, Pekow PS, Rothberg MB, Hill NS. Outcomes associated with invasive and noninvasive ventilation among patients hospitalized with exacerbations of chronic obstructive pulmonary disease. JAMA Intern Med. 2014;174:1982-1993.

23. Risom MB, Kjaer BN, Risom E, Guldager H. Non-invasive ventilation is less efficient in pneumonia than in chronic obstructive pulmonary disease exacerbation. Dan Med J. 2014;61:A4799.

24. Khalid I, Sherbini N, Qushmaq I, et al. Outcomes of patients treated with noninvasive ventilation by a medical emergency team on the wards. Respir Care. 2014;59:186-192.

25. Siemieniuk RA, Meade MO, Alonso-Coello P, et al. Corticosteroid therapy for patients hospitalized with community-acquired pneumonia: a systematic review and meta-analysis. Ann Intern Med. 2015;163: 519-528.
26. Alía I, de la Cal MA, Esteban A, et al. Efficacy of corticosteroid therapy in patients with an acute exacerbation of chronic obstructive pulmonary disease receiving ventilatory support. Arch Intern Med. 2011; 171:1939-1946.

27. Abroug F, Ouanes I, Abroug S, et al. Systemic corticosteroids in acute exacerbation of COPD: a meta-analysis of controlled studies with emphasis on ICU patients. Ann Intensive Care. 2014;4:32.

28. Ucgun I, Metintas M, Moral H, Alatas F, Yildirim H, Erginel S. Predictors of hospital outcome and intubation in COPD patients admitted to the respiratory ICU for acute hypercapnic respiratory failure. Respir Med. 2006;100:66-74.

\section{Publish your work in this journal}

The International Journal of COPD is an international, peer-reviewed journal of therapeutics and pharmacology focusing on concise rapid reporting of clinical studies and reviews in COPD. Special focus is given to the pathophysiological processes underlying the disease, intervention programs, patient focused education, and self management protocols.

\section{Dovepress}

This journal is indexed on PubMed Central, MedLine and CAS. The manuscript management system is completely online and includes a very quick and fair peer-review system, which is all easy to use. Visit http://www.dovepress.com/testimonials.php to read real quotes from published authors.

Submit your manuscript here: http://www.dovepress.com/international-journal-of-chronic-obstructive-pulmonary-disease-journal 